\title{
Priming of semantic classifications: Late and response related, or earlier and more central?
}

\author{
KARL CHRISTOPH KLAUER \\ Albert-Ludwigs-Universität Freiburg, Freiburg, Germany \\ JOCHEN MUSCH \\ Universität Mannheim, Mannheim, Germany \\ and \\ ANDREAS B. EDER \\ Rheinische Friedrich-Wilhelms-Universität Bonn, Bonn, Germany
}

\begin{abstract}
Priming of semantic classifications may occur because of response-related priming or because of priming at a more central locus. To separate these two possibilities, we randomly intermixed adjectives and first names, using a response window procedure. Participants decided whether the adjectives were positively or negatively valenced and whether the names were male or female. Each of these kinds of targets was preceded by adjective or name primes associated with responses that either matched or mismatched the correct response to the target. Results showed that priming of semantic classification involves two components: a major response-related one, modulated by prime visibility and prime-target repetition, and a smaller component with a more central locus that is less susceptible to context effects.
\end{abstract}

The classification of a target word is affected by the category of an immediately preceding prime word. For example, in affective priming (Klauer \& Musch, 2003), target words are to be classified as either evaluatively positive or negative in the so-called evaluative decision task. Evaluative decisions are faster and more accurate for target words preceded by evaluatively congruent prime words (e.g., luck-sunshine) than for target words preceded by evaluatively incongruent prime words (e.g., angersunshine). We will use the general term classification priming for priming effects in semantic classification tasks that arise as a consequence of the prime and the target being members of the same response category (congruent prime-target pairs) versus different response categories (incongruent prime-target pairs).

Classification priming can reflect facilitation and/or inhibition at the response selection stage, at the stage of categorizing targets, or during encoding or lexical access for targets. For example, according to Kunde, Kiesel, and Hoffmann (2003), participants specify so-called action triggers in elaborating the task instructions and as a consequence of practice in the classification task. These action triggers are templates against which target stim-

The research reported in this article was supported by Grant K1 614/4-4 from the Deutsche Forschungsgemeinschaft to the first author. Correspondence concerning this article should be addressed to K. C. Klauer, Institut für Psychologie, Albert-Ludwigs-Universität Freiburg, D-79085 Freiburg, Germany (e-mail: christoph.klauer@psychologie.uni-freiburg.de). uli are matched perceptually. In the case of a match, the response belonging to the matching action trigger is released. Primes have the power to bias responses if they match one of the action triggers perceptually. This account is consistent with the idea that the prime and the target are processed independently up to a response selection stage at which prime-derived and target-derived response implications interact.

Furthermore, a large literature on semantic priming suggests that prime words can facilitate encoding or lexical access for semantically related targets, including categorically related targets. A third possibility is that primederived and target-derived bits of information interact at the stage of categorizing the target in one of the response categories. Specifically, a prime word might activate the mental representation of its task-relevant category (e.g., the category positive in the evaluative decision task), thereby facilitating the categorization of congruent targets as exemplars of this same category or hindering the categorization of incongruent targets as exemplars of the other category (e.g., the category negative). We will refer to priming effects reflecting facilitation and/or inhibition of encoding, lexical access, or categorization for targets as central priming.

The purpose of the present study was to tease apart the relative contributions of response-related and central priming effects through the use of a modified priming task. We mixed two semantic classification tasks in random sequence: a gender decision task, in which the gender (male or female) of common first names had to be deter- 
mined, and the above-described evaluative decision task applied to evaluatively polarized adjectives. The kind of target (first name vs. adjective) signaled which task was to be performed on any given trial. The same response keys were used for both tasks. Kind of target and kind of prime were crossed orthogonally. This led to four different kinds of prime-target pairs (see Table 1 for examples): (1) same-response, same-task pairs, in which the prime word and the target word were mapped upon the same response via the same task; (2) different-responses, sametask pairs, in which the prime and the target were mapped upon different responses via the same task; (3) sameresponse, different-tasks pairs, in which the prime and the target were mapped upon the same response via different tasks; and (4) different-responses, different-tasks pairs, in which the prime and the target were mapped upon different responses via different tasks.

Separate indices were computed for the effects of response-related priming and central priming (see Table 1). Response-related priming should be independent of the particular task under which response-related implications of the prime and the target are derived. Its strength can therefore be evaluated on the basis of prime-target pairs with different tasks for the prime and the target. Specifically, it is assessed by the improvement in performance for same-response, different-tasks pairs, relative to different-responses, different-tasks pairs. Accordingly, response-related priming is the size of the congruency effect for different-tasks pairs.

For same-task pairs, the prime and the target additionally belong to the same category (e.g., both may be positive adjectives) or to opposite categories (e.g., one positive and one negative adjective). In the first case, primes might directly bias the correct categorization of the target (central facilitation); in the latter case, primes might hinder the correct categorization (central inhibition); both of these effects add to the response-related effects of primes just described (see Table 1). As is shown in Table 1, the congruency effect for same-task pairs should thereby exceed that for different-tasks pairs. The congruency effect for same-task pairs thus sums response-related and central priming. Subtracting response-related priming yields the index for central priming (see Table 1).

Because we mixed two semantic classification tasks, an evaluative and a gender decision task, task set priming might also play a role. Adjective targets require evaluative decisions, name targets gender decisions. Adjective primes might, therefore, prime the set of processes and task settings associated with evaluative decisions; name primes might prime those associated with gender decisions. By definition, task set priming should elevate performance for both types of same-task pairs in parallel (i.e., for same-response, same-task pairs as well as for different-responses, same-task pairs). Task set priming, if it occurs, is therefore orthogonal to (1) the index of response-related priming that relies only on differenttasks pairs, as well as to (2) the index of central priming that relies on the different-tasks pairs and the contrast between the two types of same-task pairs.

In the present study, a response window procedure (Greenwald, Draine, \& Abrams, 1996) was used that instructed the participants to give rapid responses and obliged them to maintain low variability of latency across trials. The speed pressure of the response window procedure produced a relatively high error rate. Priming effects were then observed in the accuracy domain.

Table 1

Examples of the Different Kinds of Prime-Target Pairs and Possible Effects of Primes in Each Type of Trial

\begin{tabular}{|c|c|c|c|c|}
\hline & \multicolumn{2}{|c|}{ Same Task } & \multicolumn{2}{|c|}{ Different Tasks } \\
\hline & $\begin{array}{c}\text { Same } \\
\text { Response }\end{array}$ & $\begin{array}{c}\text { Different } \\
\text { Responses }\end{array}$ & $\begin{array}{c}\text { Same } \\
\text { Response }\end{array}$ & $\begin{array}{l}\text { Different } \\
\text { Responses }\end{array}$ \\
\hline & \multicolumn{4}{|c|}{ Examples } \\
\hline & $\begin{array}{l}\text { (happy tender) } \\
\text { (Ann Mary) }\end{array}$ & $\begin{array}{l}\text { (happy cold) } \\
\text { (Ann Charles) }\end{array}$ & $\begin{array}{l}\text { (happy Mary)* }^{*} \\
\text { (Charles cold) }^{*}\end{array}$ & $\begin{array}{l}\text { (happy Charles)* } \\
\text { (Charles tender) }^{*}\end{array}$ \\
\hline & & Possible E & cts of Primes & \\
\hline \multirow[t]{3}{*}{$\begin{array}{c}\text { Response-related } \\
\text { effects } \\
+ \\
\text { Central } \\
\text { effects }\end{array}$} & $\begin{array}{c}\text { Resp.-related } \\
\text { facilitation } \\
+ \\
\text { Central } \\
\text { facilitation }\end{array}$ & $\begin{array}{c}\text { - Resp.-related } \\
\text { inhibition }^{\dagger} \\
+ \\
\text {-Central } \\
\text { inhibition }^{\dagger}\end{array}$ & $\begin{array}{l}\text { Resp.-related } \\
\text { facilitation }\end{array}$ & $\begin{array}{l}\text { - Resp.-related } \\
\text { inhibition }^{\dagger}\end{array}$ \\
\hline & \multicolumn{4}{|c|}{ Trial-Type Number } \\
\hline & (1) & (2) & (3) & (4) \\
\hline
\end{tabular}

Note- Response-related priming $=$ performance in (3) - performance in (4); central priming $=$ $(1)-(2)-[(3)-(4)]$; central facilitation $=(1)-(3)$; central inhibition $=(4)-(2)$. Resp., response; see the text. $\quad$ *In the examples, it is assumed that positive adjectives and female names are mapped on the same response key, and negative adjectives and male names on the other key. In the experiment, response mappings were counterbalanced across participants. †Inhibitory components carry a minus sign because they depress performance. 
Two factors were manipulated between participants to determine whether they dissociate response-related from central priming: First, the amount of repetition of stimulus words was varied by manipulating the set size of word pools from which primes and targets were sampled (5 words vs. 70 words). As set size decreased, the same words were more often presented as targets and thereby received more practice in being classified, which has been found necessary for these words to function as effective masked primes (e.g., Abrams \& Greenwald, 2000). Second, the visibility of the prime words (masked vs. unmasked) was manipulated to determine whether there are qualitative differences between these two modes of presentation.

\section{METHOD}

\section{Participants}

The participants were 192 University of Bonn students (96 of them male, 96 female) with different majors and nonstudent volunteers of a similar age range. All the participants were native speakers of German and had normal or corrected-to-normal vision. Two participants were excluded from the analyses because they did not follow the instructions and used only one response key throughout the experiment.

\section{Design}

The experimental design was a balanced 2 (repetition) $\times 2$ (prime visibility) $\times 4$ (kind of prime-target pairs) design, kind of primetarget pairs being a within-participants factor. The two response keys were to be pressed with different hands. Assignment of positive adjectives to the dominant versus nondominant hand and assignment of female first names to the dominant versus nondominant hand were counterbalanced with the experimental factors of repetition and prime visibility; all of these factors were between-participants factors. Six male and 6 female participants were assigned to each group defined by a combination of the factors above.

\section{List Construction}

The 70 male and 70 female first names, as well as the 70 positive and 70 negative adjectives, were the same as those used by Klauer and Musch (2002). For each participant, a new list of prime-target pairs was constructed, consisting of blocks of 48 prime-target pairs each. The 48 trials realized a balanced 4 (type of prime: positive adjective, negative adjective, male first name, or female first name) $\times$ 4 (type of target) design. The primes and targets were randomly drawn with replacement either from the word pools above, comprising 70 words of each type (low repetition), or from small word pools of 5 words of each kind (high repetition). New small word pools were randomly sampled from the large word pools for each participant in the high-repetition groups.

In these groups, each individual stimulus word was thereby expected to appear, on average, 2.4 times as target and 2.4 times as prime per block of 48 trials. In the low-repetition groups, these numbers were 0.17 and 0.17 , respectively.

\section{Response Window Procedure and Presentation Parameters}

The participants were seated at a distance of $50 \mathrm{~cm}$ from a $43-\mathrm{cm}$ VGA color monitor with a $70-\mathrm{Hz}$ refresh rate. Stimulus presentation and measurement of response latencies were controlled by a software timer with video synchronization and millisecond accuracy (Haussmann, 1992).

The sequence of events on masked priming trials was as follows: a forward mask for $300 \mathrm{msec}$, the prime for $57 \mathrm{msec}$, a backward mask for $14 \mathrm{msec}$, then the target. The masks were letter strings of
13 randomly sampled consonants. The masks, primes, and targets were presented in black on a light gray background centered on the middle of the screen. The primes were extended to a length of 13 letters by adding random consonants to the left and right. For example, the prime word brave thereby became gkvfbravemltr. New random consonants were sampled for each different prime word for each participant.

The participants had a window of 144-msec duration, initially centered at $500 \mathrm{msec}$ after target onset, within which they were to respond to the target stimulus. Onset and offset of the response window, as well as feedback on whether the response had occurred within the response window, were signaled by changes of the target color. Following the response, target offset occurred after $300 \mathrm{msec}$. The next trial was then initiated after an interval of $150 \mathrm{msec}$. All the stimuli were presented in lowercase letters.

To tailor the window center to each participant's performance, an adaptive procedure was used. Following Greenwald et al. (1996), the window center was adjusted after each block of 48 trials, contingent on the participant's performance in that block. It was decreased by $28 \mathrm{msec}$ if, in that block, there were no more than $20 \%$ errors and the mean response latency did not exceed the window center by more than $100 \mathrm{msec}$. It was increased by $28 \mathrm{msec}$ if there were $45 \%$ or more errors and the mean response latency exceeded the window center by more than $100 \mathrm{msec}$. If neither of these sets of conditions was met, the window center was not changed.

The trials in the group with visible primes differed in that (1) forward and backward masks were replaced by blanks and (2) prime words were presented without flanking random consonants.

The participants completed 10 experimental blocks preceded by 3-10 practice blocks. The participants performed a minimum of 3 practice blocks. They continued practice until no further adaptation of the response window center occurred or until a maximum number of 10 practice blocks had been performed.

\section{Direct Measure of Prime Visibility}

In the groups with masked primes, additional blocks of 48 primetarget pairs were presented after the experimental blocks for a test of prime visibility. To maximize the sensitivity of the test, each test block presented either only adjectives or only first names as primes and targets. This allowed the participants to focus on perceiving either the masked adjectives' valences or the masked first names' genders throughout each block. The words were sampled from the pools used for the participant's experimental trials. The sequence and timing of the events in the visibility test trials were the same (including the presentation of target words) as those in the experimental trials.

The participants were instructed to decide whether the prime words were positive or negative for adjective blocks and whether the prime words were male or female for name blocks; thus, the participants' task was semantic classification of the primes, rather than the targets. The order of the two kinds of blocks was completely crossed with the experimental factor of repetition and with the other counterbalancing factors (see the Design section). The participants were no longer required to respond within the response window, since time pressure would decrease the sensitivity of the direct test.

For each kind of material (adjectives and first names), the participants first underwent three practice blocks, followed by two data collection blocks. In the first practice block, masks were omitted, and the primes were colored in red. The second block added masks; in the third practice block, the prime display reverted to normal black, as in the experimental trials.

\section{RESULTS}

\section{Priming Effects}

Responses with latencies below $100 \mathrm{msec}$ or above $1,000 \mathrm{msec}$ were excluded from the analyses, thereby re- 
Table 2

Percentages of Correct Responses and Mean Correct Response Latencies (in Milliseconds) for the Different Kinds of Trials (With Standard Deviations)

\begin{tabular}{|c|c|c|c|c|c|c|c|c|c|c|c|}
\hline \multirow{3}{*}{$\begin{array}{c}\text { Prime } \\
\text { Visibility }\end{array}$} & \multirow{3}{*}{$\begin{array}{l}\text { Set } \\
\text { Size }\end{array}$} & \multicolumn{5}{|c|}{ Same Task } & \multicolumn{5}{|c|}{ Different Tasks } \\
\hline & & \multicolumn{2}{|c|}{$\begin{array}{c}\text { Same } \\
\text { Response }\end{array}$} & \multicolumn{2}{|c|}{$\begin{array}{c}\text { Different } \\
\text { Responses }\end{array}$} & \multirow{2}{*}{$\begin{array}{c}\mathrm{RC} \\
\text { Effect }\end{array}$} & \multicolumn{2}{|c|}{$\begin{array}{c}\text { Same } \\
\text { Response }\end{array}$} & \multicolumn{2}{|c|}{$\begin{array}{c}\text { Different } \\
\text { Responses }\end{array}$} & \multirow{2}{*}{$\begin{array}{c}\text { RC } \\
\text { Effect }\end{array}$} \\
\hline & & $M$ & $S D$ & $M$ & $S D$ & & $M$ & $S D$ & $M$ & $S D$ & \\
\hline \multicolumn{12}{|c|}{ Accuracy Data } \\
\hline Visible & Small & 81.80 & 10.06 & 70.91 & 10.09 & 10.89 & 79.00 & 10.73 & 69.88 & 9.43 & 9.12 \\
\hline \multirow{3}{*}{ Masked } & Large & 77.72 & 9.38 & 68.88 & 8.46 & 8.84 & 75.40 & 9.01 & 68.40 & 9.07 & 7.00 \\
\hline & Small & 81.20 & 6.88 & 72.76 & 8.85 & 8.44 & 78.61 & 7.88 & 72.65 & 9.30 & 5.69 \\
\hline & Large & 77.09 & 8.14 & 72.05 & 8.42 & 5.04 & 74.89 & 8.60 & 71.86 & 7.89 & 3.03 \\
\hline \multicolumn{12}{|c|}{ Latency Data } \\
\hline \multirow[t]{2}{*}{ Visible } & Small & 514 & 68 & 544 & 67 & 30 & 532 & 76 & 550 & 70 & 18 \\
\hline & Large & 535 & 57 & 565 & 58 & 30 & 552 & 60 & 570 & 56 & 18 \\
\hline \multirow[t]{2}{*}{ Masked } & Small & 501 & 65 & 518 & 61 & 17 & 506 & 66 & 518 & 63 & 12 \\
\hline & Large & 531 & 64 & 541 & 61 & 10 & 537 & 64 & 542 & 62 & 5 \\
\hline
\end{tabular}

Note-RC effect, absolute magnitude of the response compatibility effect.

moving $2.0 \%$ of the data. Table 2 presents the percentages of correct responses for the different kinds of prime-target pairs, along with the mean latencies of correct responses. ${ }^{1}$ Two contrasts were computed over the different kinds of prime-target pairs to define the two dependent variables, response-related and central priming effects, as explained in the introduction and Table 1. Figure 1 shows responserelated and central priming.

Note that because the subsequent analyses were performed on priming effects, rather than treating priming as a separate factor in the analyses, a main effect actually represents an interaction with priming and interactions represent higher order interactions with priming. In an ANOVA with repetition and prime visibility as factors, response-related priming was significantly larger than zero $[F(1,186)=108.92, p<.01]$. There were significant main effects of repetition $[F(1,186)=4.41, p<.05]$ and of prime visibility $[F(1,186)=8.77, p<.01]$ but no significant interaction of the two $(F<1)$. In an ANOVA with the same factors, central priming was significantly larger than zero $[F(1,186)=12.07, p<.01]$, but none of the other effects approached significance (all $F_{\mathrm{S}}<1$ ).

Thus, practice in classifying words as targets increases response-related priming effects, as evidenced by the
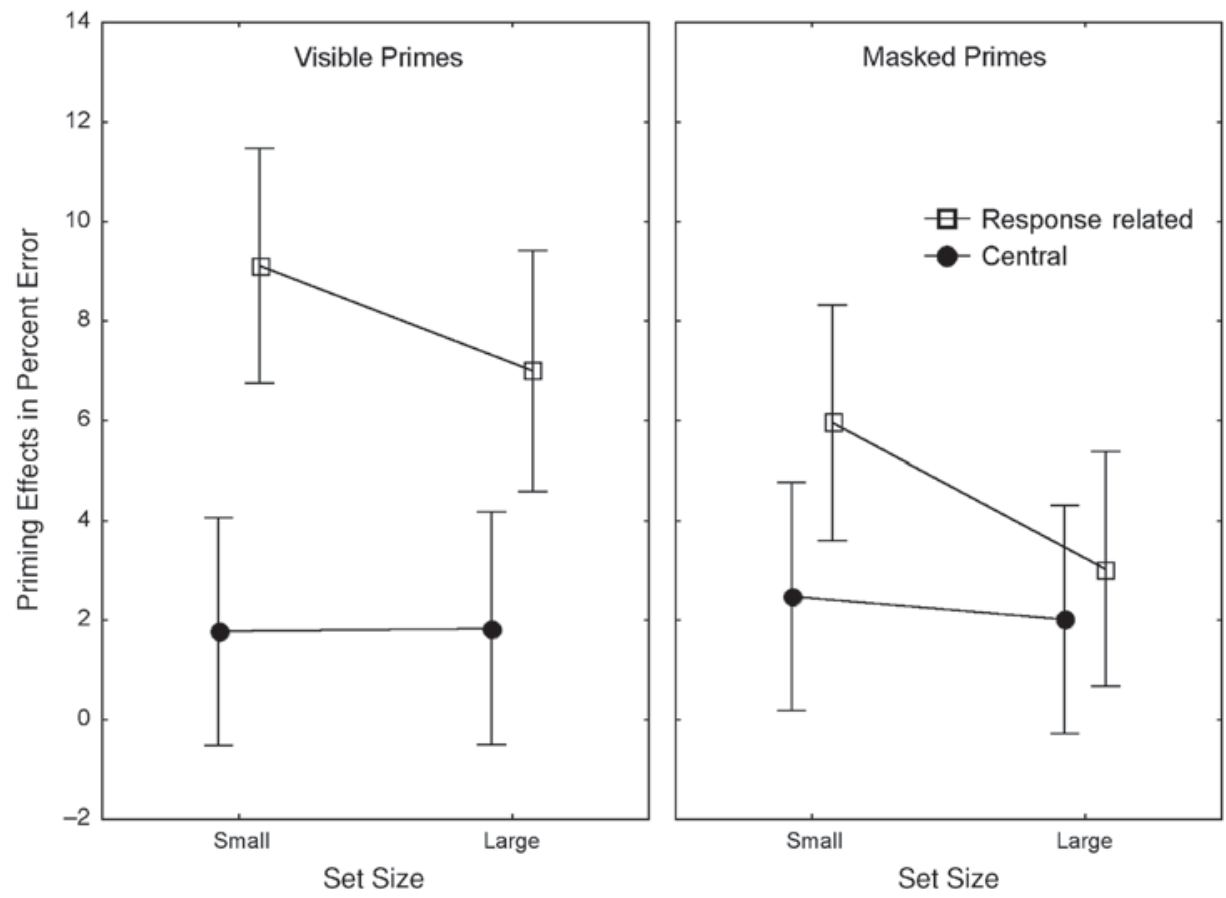

Figure 1. Central and response-related priming effects for visible primes (left panel) and masked primes (right panel) as functions of set size. Error bars indicate $95 \%$ confidence intervals. 
repetition effect. Furthermore, visible primes engender larger priming effects than do masked primes in responserelated priming. However, even when masked primes and low repetition were given, response-related priming was still significantly larger than zero $[M=3.03, S D=6.07$; $t(47)=3.45, p<.01]$.

In contrast, central priming was smaller and was not affected by repetition or prime visibility. Note for later reference that central priming was significant in masked priming $[M=2.25, S D=8.23 ; t(95)=2.68, p<.01] .^{2}$

A joint ANOVA with locus of priming (central vs. response related) as an additional factor revealed that central priming $(M=2.02, S D=7.98)$ was significantly smaller than response-related priming $[M=6.27, S D=$ $8.51 ; F(1,186)=17.22, p<.01]$ and that the interaction of locus of priming and prime visibility approached significance $[F(1,186)=3.82, p=.052]$. The interaction of locus of priming and repetition, although apparent in the simple effects analyses above, was not significant $[F(1,186)=1.29, p=.26] .^{3}$

Whereas a cost-benefit analysis for response-related priming is not possible in the absence of neutral primes, a cost-benefit analysis of central priming is possible. For example, as is shown in Table 1, in same-response, sametask pairs, primes can engender both response-related and central facilitation; in same-response, different-tasks pairs, they can engender only response-related facilitation; hence, central facilitation is given by the difference in performance between the two types of prime-target pairs. Table 3 shows the indices for central facilitation and central inhibition as defined in Table 1 for each combination of prime visibility and repetition. These are signed so that positive values indicate a positive contribution to the overall central priming effect. An ANOVA with kind of central priming (facilitation vs. inhibition), repetition, and prime visibility as factors revealed that facilitation $(M=$ $2.48, S D=5.66)$ outweighed inhibition $[M=-0.45$, $S D=5.43 ; F(1,186)=27.16, p<.01]$. No other effect approached significance (all $F \mathrm{~s}<1$ ). Note that the balance of central facilitation and inhibition, but not overall central priming, would be shifted by possible task set priming (see the introduction), so this cost-benefit analysis should be interpreted with caution.

Direct measure of prime visibility. The direct test of prime visibility revealed mean $d^{\prime}$ values of $0.03(S D=$ $0.30)$ and $0.00(S D=0.31)$ for the participants' abilities

Table 3

Central Facilitation and Inhibition as a Function of Prime Visibility and Set Size (With Standard Deviations)

\begin{tabular}{ccccccc}
\hline \multirow{2}{*}{$\begin{array}{c}\text { Prime } \\
\text { Visibility }\end{array}$} & Set & \multicolumn{2}{c}{ Facilitation } & & \multicolumn{2}{c}{ Inhibition } \\
\cline { 7 - 7 } \cline { 6 - 7 } & Size & $M$ & $S D$ & & $M$ & $S D$ \\
\hline Visible & Small & 2.80 & 5.94 & & -1.03 & 4.63 \\
& Large & 2.32 & 5.21 & & -0.48 & 5.86 \\
Masked & Small & 2.59 & 5.17 & & -0.11 & 6.40 \\
& Large & 2.21 & 6.36 & & -0.19 & 4.79 \\
\hline
\end{tabular}

Note-Central facilitation was significant in each row in individual twotailed $t$ tests (smallest $t=2.40$, largest $p=.02$ ). Central inhibition was never significant (largest $|t|=1.54$, smallest $p=.13$ ). to discriminate the masked adjectives' valence and the masked names' gender, respectively. Both were not significantly different from zero $[t(95)=1.01, p=.31$, and $t(95)=0.00, p=.99$, respectively].${ }^{4}$ Nor were there any effects in ANOVAs of these $d^{\prime}$ values with repetition and order of direct tests (adjectives first vs. first names first) as factors (largest $F=2.27$, smallest $p=.14$ ).

\section{DISCUSSION}

There were three major findings: (1) Response-related priming was much stronger than central priming, yet (2) there was a significant amount of central priming in both masked and unmasked priming, and (3) responserelated priming was much more dependent on the context factors of prime visibility and repetition than was central priming.

Response-related priming was significantly larger than central priming. This suggests that only a relatively small proportion of classification priming effects is directly related to the congruence and incongruence of the categories of the prime and the target; the major part appears to be due to interactions in the independent response tendencies triggered by the prime and the target. Mixing two semantic classification tasks allowed us to disentangle the two different contributions.

Response-related priming is consistent with the recent account in Kunde et al. (2003), briefly sketched in the introduction. Whether the prime and the target share the same dimension of categorization (i.e., valence or gender) or not is irrelevant in this account. Yet there was a significant amount of central priming even in groups with masked primes: Congruency effects were consistently larger by a small amount when they involved primes and targets from the same dimension of categorization (e.g., positive and negative words), as compared with when they involved primes and targets from unrelated, or perhaps more cautiously phrased, less strongly related categories (e.g., positive words and male names). The account in Kunde et al. cannot explain central priming without modification. Central priming suggests that primes can facilitate or inhibit the classification process for targets from the same dimension of categorization. For example, according to Masson and Bodner (2003), the similarity of the processing operations applied to a prime event and those applied to a target determine the extent to which target processing is affected by the prime.

There were no qualitative differences between masked and visible priming. There were, however, qualitative differences between response-related and central priming. Response-related priming was a function of repetition and prime visibility, whereas central priming was not affected by these factors.

As repetition increases, the same words are more often presented as targets and, thereby, receive more practice in being classified. Such practice has been found necessary for these words to function as effective masked primes (e.g., Abrams \& Greenwald, 2000). Repetition effects were restricted to response-related priming, suggest- 
ing that practice effects in classification priming act at the level of response-related decisions. ${ }^{5}$ For example, in Kunde et al.'s (2003) model, action triggers are adapted, or newly formed, to match words previously seen as targets in the course of practice. The model in Kunde et al. can also account for the effect of prime visibility in responserelated priming: It is plausible that a perceptual match with an action trigger is less likely to occur for stimuli degraded by masks than for clearly visible stimuli.

It is unlikely, although not impossible, that central priming reflects facilitation in encoding or lexical access for targets, because there is little evidence for priming by irrelevant categorical relationships between the prime and the target in classification priming (Klauer \& Musch, 2002, 2003). For example, there is little effect of whether or not both the prime and the target refer to animate objects in the evaluative decision task. We propose instead that central priming reflects facilitation in categorizing targets. In this analysis, the task set for target processing is applied to primes (Dehaene et al., 1998), leading to activation of the prime's task-relevant category (e.g., the category positive in the evaluative decision task). This preactivation helps in the categorization of a target of that same category, leading to central priming for such targets.

Because we mixed two tasks, an evaluative decision task and a gender decision task, task set priming may also play a role, elevating performance for both kinds of sametask pairs (i.e., for same-response, as well as for differentresponses, prime-target pairs). As was explained in the introduction, the present estimate of central priming is orthogonal to a possible task set priming effect, but the separate estimates of central facilitation and inhibition cannot be disentangled from task set priming. Suppose that instead of evaluative decisions and gender decisions, we had mixed two different tasks, Tasks 1 and 2. For Task 1 , only positive adjectives and female (male) names are presented and assigned to response keys 1 and 2, respectively. For Task 2, male (female) names and negative adjectives are assigned to response keys 1 and 2, respectively. Both tasks, relying on arbitrary mappings, should be well practiced individually before being presented in an intermixed fashion. Mixing the tasks yields the same stimulus-response mappings as when the evaluative decision task and a gender decision task are mixed. However, when Tasks 1 and 2 are mixed and central priming is computed precisely as before, task set priming is no longer orthogonal to central priming, which is not difficult to see. Comparing the new experimental condition with the one realized here thereby allows one to estimate the impact of task set priming and to disentangle its possible contributions from the estimates of central facilitation and inhibition (see also note 3 ).

According to the present findings, classification priming involves two components: a major response-related one, modulated by context factors such as repetition and prime visibility, and a smaller one with a more central locus that is less susceptible to context effects. The former component can be readily accommodated by exist- ing accounts that postulate independent processing of the prime and the target up to a late level of response-related decisions. The latter component suggests that prime and target processing already interact at an earlier stage of processing.

\section{REFERENCES}

Abrams, R. L., \& Greenwald, A. G. (2000). Parts outweigh the whole (word) in unconscious analysis of meaning. Psychological Science, 11, 118-124.

Dehaene, S., Naccache, L., LeClec'H, G., Koechlin, E., Mueller, M., Dehaene-Lambertz, G., ET AL. (1998). Imaging unconscious semantic priming. Nature, 395, 597-600.

Greenwald, A. G., Draine, S. C., \& Abrams, R. L. (1996). Three cognitive markers of unconscious semantic activation. Science, $\mathbf{2 7 3}$, 1699-1702.

Greenwald, A. G., Klinger, M. R., \& Schuh, E. (1995). Activation by marginally perceptible ("subliminal") stimuli: Dissociation of unconscious from conscious cognition. Journal of Experimental Psychology: General, 124, 22-42.

Greenwald, A. G., McGhee, D. E., \& Schwartz, J. L. K. (1998). Measuring individual differences in implicit cognition: The implicit association test. Journal of Personality \& Social Psychology, 74, 1464-1480.

Haussmann, R. E. (1992). Tachistoscopic presentation and millisecond timing on the IBM PC/XT/AT and PS/2: A Turbo Pascal unit to provide general-purpose routines for CGA, Hercules, EGA, and VGA monitors. Behavior Research Methods, Instruments, \& Computers, 24, 303-310.

Klauer, K. C., Draine, S. C., \& Greenwald, A. G. (1998). An unbiased errors-in-variables approach to detect unconscious cognition. British Journal of Mathematical \& Statistical Psychology, 51, 253267.

Klauer, K. C., \& Musch, J. (2002). Goal-dependent and goal-independent effects of irrelevant evaluations. Personality \& Social Psychology Bulletin, 28, 802-814.

Klauer, K. C., \& Musch, J. (2003). Affective priming: Findings and theories. In J. Musch \& K. C. Klauer (Eds.), The psychology of evaluation: Affective processes in cognition and emotion (pp. 7-50). Mahwah, NJ: Erlbaum.

Kunde, W., Kiesel, A., \& Hoffmann, J. (2003). Conscious control over the content of unconscious cognition. Cognition, 88, 223-242.

Masson, M. E. J., \& Bodner, G. E. (2003). A retrospective view of masked priming: Toward a unified account of masked and long-term repetition priming. In S. Kinoshita \& S. J. Lupker (Eds.), Masked priming: The state of the art (pp. 57-94). New York: Psychology Press.

Mayr, U., \& Keele, S. W. (2000). Changing internal constraints on action: The role of backward inhibition. Journal of Experimental Psychology: General, 129, 4-26.

\section{NOTES}

1. Analyses of response latencies revealed a pattern of effects analogous to the one in the accuracy domain. The results, therefore, do not reflect speed-accuracy trade-offs.

2 . The effect remained significant in the regression analysis proposed by Greenwald, Klinger, and Schuh (1995), in which the priming effect is regressed on the direct measure of prime visibility (i.e., on the mean of the two $d^{\prime}$ values for masked adjectives and masked names), and in the improved version of it proposed by Klauer, Draine, and Greenwald (1998). This was also true of masked response-related priming.

3. Control analyses were run to determine whether response key mapping or task switching affected response-related or central priming. There were two qualitatively different response mappings: either male and negative shared the same response key and female and positive the other response key, or male and positive shared the same response key and female and negative the other response key. In studies in which the implicit association test was used (Greenwald, McGhee, \& Schwartz, 
1998), the first response mapping sometimes led to faster and more accurate responding than did the second response mapping, especially among female participants. This is interpreted as evidence for a stronger association of female with positive than with negative. Cross-task associations of this kind might modulate priming effects. To consider task switching, the priming trials required frequent switches between the evaluative decision task and the gender decision task. Task switching has been argued to involve rapid activation of the appropriate task set and inhibition of the inappropriate task set (e.g., Mayr \& Keele, 2000); these processes leave their traces in performance differences between trials involving the same task as the previous trial and trials involving a task switch. The control analyses did not reveal effects of response mapping or task switching that interacted with the present priming effects. Details of these analyses can be obtained from the first author.

4. It could be argued that the direct-test trials would have been more comparable to the priming trials if adjectives and first names had been used in an intermixed, rather than a blocked, fashion. However, doing so might have added to the participants' confusion in an already difficult task.

5. As repetition increases, the proportion of prime-target pairs with identical primes and targets increases. Response-related priming is, however, assessed on the basis of primes and targets from different categories. The repetition effect in response-related priming cannot, therefore, be explained by possible repetition priming. In addition, excluding trials with identical primes and targets did not change the pattern of effects in any of the analyses above.

(Manuscript received May 17, 2004; revision accepted for publication December 14, 2004.) 